\title{
Research Paper: Effects of Upper Limb Resistance Training on Cognition and Daily Living Activities in Older Adults
}

\author{
Navid Mirzakhani' ${ }^{1}$ (D), Habib Esmailian² ${ }^{2}$ Ali Asghar Jamebozorgi ${ }^{3}$, , Seyed Mehdi Tabatabaee $^{3}$ (D), Mahnaz Hejazi-Shirmard ${ }^{1,4^{*}}$ (i)
}

1. Department of Occupational Therapy, School of Rehabilitation, Shahid Beheshti University of Medical Sciences, Tehran, Iran.

2. Physical Medicine and Rehabilitation Research Center, Shohada-e-Tajrish Hospital, Shahid Beheshti University of Medical Sciences, Tehran, Iran.

3. Physiotherapy Research Center, School of Rehabilitation, Shahid Beheshti University of Medical Sciences, Tehran, Iran.

4. Department of Occupational Therapy, School of Rehabilitation Sciences, Iran University of Medical Sciences, Tehran, Iran.

\begin{tabular}{|c|c|}
\hline $\begin{array}{l}\text { Use your device to scan } \\
\text { and read the article online }\end{array}$ & Citation Mirzakhani N, Esmailian H, Jamebozorgi AS, Tabatabaei SM, Hejazi Shirmard M. Effects of Upper Limb Re- \\
\hline 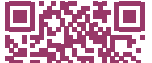 & $\begin{array}{l}\text { sistance Training on Cognition and Daily Living Activities in Older Adults Iranian Rehabilitation Journal. 2022; 20(Special } \\
\text { Issue):47-54. http://dx.doi.org/10.32598/irj.20.SpecialIssue.1436.1 }\end{array}$ \\
\hline 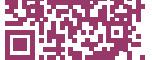 & doi'http://dx.doi.org/10.32598/irj.20.SpecialIssue.1436.1 \\
\hline
\end{tabular}

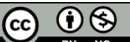

Article info:

Received: 14 Jul 2021

Accepted: 06 Oct 2021

Available Online: 01 Jan 2022

\section{Keywords:}

Elderly, Resistance training, Upper limb, Cognition, Daily living activities

\section{A BSTRACT}

Objectives: Physical exercise is a promising intervention to improve cognitive function and reduce the risk of dementia and other related neurodegenerative disorders. The present study aimed to investigate the effectiveness of an upper limb-focused physical exercise intervention on cognitive function and daily living activities in older adults.

Methods: Forty older residents of a nursing home participated in this clinical trial and were randomly assigned to the experimental $(\mathrm{n}=20)$ or the control $(\mathrm{n}=20)$ groups. Participants in the experimental group received a physical exercise intervention for six weeks. This intervention included resistance training of the upper limbs using free weights. Evaluations were performed at baseline and after three and six weeks of the resistance training. Stroop test and Barthel index were used to evaluate cognitive function and daily living activities, respectively.

Results: Upper limb resistance training significantly improved cognitive function in the experimental group, but there was no significant difference between the groups in the ability to perform daily activities.

Discussion: The present study revealed that resistance training of the upper limb can yield cognitive improvement in older adults. Upper limb strengthening seems to have the potential to improve cognitive performance in the elderly and can be recommended as a regular exercise activity. However, further studies with more comprehensive outcome measures are needed.

\section{* Corresponding Author:}

Mahnaz, Hejazi Shirmard, PhD.

Address: Department of Occupational Therapy, School of Rehabilitation Sciences, Shahid Beheshti University of Medical Sciences, Tehran, Iran. Tel: +98 (21) 77561721

E-mail: Hejazishirmard.m.@sbmu.ac.ir ; m.hejazishirmard@yahoo.com 


\section{Highlights}

- Normal aging is associated with the inevitable deterioration of cognitive function.

- Cognitive deficits in older adults negatively affect their ability to participate in activities of daily living.

- Upper limb-focused resistance training may be an effective intervention to promote cognitive function in the elderly.

- There is a need for further clinical trials with more responsive outcome measures to investigate the effectiveness of upper limb-focused resistance training on daily living activities in the elderly.

\section{Plain Language Summary}

Aging is a degenerative process characterized by physical and cognitive decline. Recently, the positive effects of physical exercise, including aerobic and resistance training, on the physical and cognitive performance of the elderly have been well established. Considering that the cognitive function and motor function of the upper limb are closely related, the present study investigated the effects of a 6-week upper limb-focused resistance training on cognitive function and also on daily living activities in older adults. The findings showed that upper limb resistance training can promote cognitive function in the elderly, but it did not change the ability to accomplish daily living activities.

\section{Introduction}

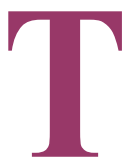

he world is aging and the number of elderly people over 60 is increasing [1]. Aging is a dynamic process and is accompanied by structural and functional changes in body systems $[1,2]$. Changes in sensory-motor, cognitive and psychosocial performance are inevitable with age. Normal aging is associated with a decrease in white matter and gray matter volume in the hippocampus and amygdala, as well as in the frontal, temporal, parietal, and occipital cortices. Because these areas have essential roles in cognitive functions, their degeneration is often associated with cognitive decline; thus, older people may experience a variety of cognitive deficits, including problems with attention, working and long-term memory, problem-solving skills, information-processing speed, and reasoning [2,3]. Cognitive deficits jeopardize the elderly's ability to plan and sequence, and negatively impact their participation in activities of daily living, work, and leisure [2]. Consequently, these deficits may lead to dependency in daily activities, disability and, low quality of life [3].

Physical inactivity is one of the main predictors of cognitive decline in older adults [4]. Although lifelong participation in physical activity is recommended, adopting an active lifestyle at any age is advantageous for delaying or reducing cognitive decline [5]. It is hypothesized that physical exercise increases the volumes of the prefrontal cortex and the anterior hippocampus, enhances neurogenesis, angiogenesis, and synaptic plasticity, reduces the pro-inflammatory process, decreases cell damage due to oxidative stress, and consequently improves cognitive function $[5,6]$.

The physical and cognitive benefits of physical exercise in older adults have been well established in recent studies $[5,7]$ and the effectiveness of aerobic exercise has been mainly studied and resistance exercise has rarely been investigated [8]. On the other hand, recent studies have indicated that cognitive deterioration is associated with impaired physical function $[9,10]$. However, there is also little evidence on the relationship between cognitive function and upper limb function $[9,10]$. During normal aging and early stages of Alzheimer's disease, the frontal lobe, especially the frontoparietal and frontostriatal areas are affected. These areas are involved in higher-level cognitive functions [11, 12]. Some studies have suggested that these areas are also active during motor control, gross and fine motor activities of the upper limb [11, 12]. Therefore, it can be concluded that improving upper limb motor capacities may also enhance cognitive function in the elderly. To the best of our knowledge, no study has investigated the effect of upper limb resistance training on cognitive function in the elderly. Therefore, the primary aim of the present study was to investigate the effect of resistance training of the upper limb on the cognitive performance of older adults. Because cognitive function may affect the ability of older people to perform daily activities [2], another 
aim was to examine the effect of these exercises on the ability to perform daily occupations.

\section{Materials and Methods}

In this single-blinded randomized clinical trial, older residents of Shaystegan nursing home (under the supervision of the State Welfare Organization) in Shahr-e Kord were screened for inclusion criteria. Forty eligible elders were recruited for the study. The inclusion criteria included the age of $\geq 60$ years, acceptable cognitive function (score $\geq 21$ in Mini-Mental State Examination) [13], absence of cardiovascular diseases, and ability to communicate and perform the instructions. Exclusion criteria were the presence of any acute orthopedic, rheumatologic, or any other neurologic conditions that may affect the function of the upper limb. Participants were assessed and randomly assigned to two groups using a sealed-envelope randomization method.

The intervention in the experimental group consisted of 60-minute sessions and was performed three times per week, for six weeks. Each session consisted of three parts, including 10 minutes of warm-up exercise (stretching the large muscle groups of the upper limb, including triceps, deltoids, pectoralis major, and shoulder external rotators), 40 minutes of upper limb resistance training (20 minutes for each limb), and 10 minutes of cool-down exercise. The intensity of the exercises was based on eight repetitions of $60 \%$ of 1 Repetition Maximum (RM) [14]. Each RM is the maximum weight that a person can overcome for one movement. Exercises involved shoulder abduction and adduction, external and internal rotation, flexion and extension, elbow flexion and extension, forearm supination and pronation, and wrist extension and flexion. Each exercise was repeated eight times in two sets. Participants rested for 30 seconds between two sets of one exercise and 1 minute between each exercise. Intervention for the control group consisted only of stretching exercises. Dependent variables, including cognitive function and independence in daily life activities in participants, were assessed by the assessor three times, at baseline and three and six weeks after the resistance training. The assessor was a postgraduate occupational therapist with three years of clinical experience, who was blinded to the participant's group assignment.

Data gathering included socio-demographic information, cognitive screening (using Mini-Mental State Exam; MMSE), and assessment of cognitive function and activities of daily living. The elderly's cognitive function was measured using the Stroop test. Stroop is a neuropsychological test that has been widely used to evaluate the executive function, selective attention, and the ability to inhibit cognitive interference but it also measures other cognitive functions, such as processing speed, cognitive flexibility, and working memory $[15,16]$

We used the computer version of the Stroop test because it is a more accurate scale of reaction time and reduces response error [17]. This test consisted of two conditions: congruent and incongruent and each condition included 48 stimuli. Also, 48 congruent colored words (the color ink and word were the same; for example, BLUE written in blue ink) and 48 incongruent colored words (the color ink and word were different; for example, the BLUE written in red ink) were presented to each participant. The display distance between stimuli was 800 milliseconds and the stimulus display time was 2000 milliseconds [18]. Participants were asked to report the ink color of the words in both conditions. To do this, they pressed keys labeled with a color label on the computer keyboard screen in blue, red, yellow, and green. The test was performed in a quiet room to minimize distraction. After recording the personal information and before starting the test, the assessor familiarized the participant with the implementation of the test through the part, which was designed for this purpose. To interpret the results of the test, the following scores were computed separately by the software for the congruent and incongruent conditions: reaction time and the number of correct answers or the number of errors. Shorter reaction time and fewer errors in each condition are indicators of better cognitive performance.

The Barthel index is one of the most commonly used disability scales, designed by Mahoney and Barthel in 1955 [19]. Ten items of daily living activities, including feeding, grooming, bathing, dressing, bladder and bowel control, toilet use, transfer, ambulation, and stair climbing are assessed [20]. This assessment is accomplished through an interview and is scored on a 0-100 scale. Higher scores indicate more independence in daily life activities [20, 21].

The Shapiro-Wilk test confirmed the normal distribution of all variables. To evaluate the comparability of the baseline socio-demographic characteristics, independent samples t-test and Chi-square tests were performed for quantitative and qualitative variables, respectively. A two-way repeated-measures analysis of variance with a group (experiment group and control group) and time (baseline, T2, and T3) was conducted to determine the effects of exercise on cognitive function and activities of daily living. Statistical significance was set at $\mathrm{P}<0.05$. 


\section{Results}

A total of 40 eligible older adults were randomly assigned to the experimental or control groups. All participants completed the program and were evaluated at the end of the study. At baseline, there were no statistically significant differences between the two groups in key characteristics, including age and sex, MMSE score, and dependent variables, including independence in activities of daily living and cognitive function $(\mathrm{P}>0.05)$ except for reaction time and the number of errors in the incongruent condition of the Stroop test, which were higher in the experimental and control groups, respectively. Table 1 shows the results of the cognitive function and activities of daily living in two groups.

The results showed significant main effects of time on reaction time and the number of errors in the congruent condition, and reaction time of the incongruent condition of the Stroop test. Whereas the main effect of the group was significant for the number of errors in the congruent condition and for both reaction time and the number of errors in the incongruent condition. The group $\times$ time interaction was significant for both variables of the congruent condition and the reaction time of the incongruent condition (Table 2).

Finally, resistance training of the upper limb yielded significant improvement in cognitive function in the experimental group but we found no significant difference in activities of daily living between groups after the intervention.

\section{Discussion}

The effect of aerobic exercise on the cognitive function of the elderly has been studied in numerous studies, but due to the high risk of cardiovascular dysfunction and rheumatic disorders of the hip and knee, many older people cannot do these exercises [22]. The present study

Table 1. Descriptive statics of the outcome measures

\begin{tabular}{|c|c|c|c|c|c|c|c|}
\hline \multirow{3}{*}{\multicolumn{2}{|c|}{ Variables }} & \multicolumn{6}{|c|}{ Mean \pm SD } \\
\hline & & \multicolumn{3}{|c|}{ Resistance Training Group } & \multicolumn{3}{|c|}{ Control Group } \\
\hline & & T1 & T2 & T3 & T1 & T2 & T3 \\
\hline \multicolumn{8}{|c|}{ Stroop test } \\
\hline \multirow{2}{*}{$\begin{array}{l}\text { Congruent } \\
\text { task }\end{array}$} & Reaction time & $82.7 \pm 2.63$ & $81.3 \pm 2.95$ & $79.8 \pm 2.96$ & $82.3 \pm 3.1$ & $81.3 \pm 3.06$ & $81.2 \pm 3.3$ \\
\hline & Error & $12.55 \pm 4.36$ & $10.85 \pm 4.25$ & $9.80 \pm 3.79$ & $15.20 \pm 3.36$ & $14.25 \pm 3.56$ & $13.80 \pm 3.66$ \\
\hline \multirow{2}{*}{$\begin{array}{l}\text { Incongruent } \\
\text { task }\end{array}$} & Reaction time & $87.9 \pm 1.65$ & $86.2 \pm 2.19$ & $85.75 \pm 2.29$ & $85.6 \pm 2.75$ & $84.4 \pm 2.58$ & $84.1 \pm 3.3$ \\
\hline & Error & $16.40 \pm 3.69$ & $15.25 \pm 3.61$ & $14.50 \pm 3.36$ & $19.10 \pm 4.19$ & $18.10 \pm 3.98$ & $19.55 \pm 6.37$ \\
\hline \multicolumn{2}{|c|}{ Barthel index } & $55.0 \pm 18.13$ & $55.5 \pm 17.68$ & $56.5 \pm 17.25$ & $55.25 \pm 17.87$ & $55 \pm 17.91$ & $54 \pm 18.03$ \\
\hline
\end{tabular}

T1: Baseline; T2: week three, T3: week six

Пranian Rehabilitation Journal

Table 2. Analysis of variance of the outcome measures (ANOVA)

\begin{tabular}{|c|c|c|c|c|c|c|c|c|c|c|}
\hline \multirow{2}{*}{\multicolumn{2}{|c|}{ Variables }} & \multicolumn{3}{|c|}{ Time Effect } & \multicolumn{3}{|c|}{ Group Effect } & \multicolumn{3}{|c|}{ Group×Time Effect } \\
\hline & & \multirow[t]{2}{*}{$\mathbf{F}$} & \multirow[t]{2}{*}{$\mathbf{P}$} & \multirow{2}{*}{ Partial $\eta^{2}$} & \multirow[b]{2}{*}{ troop } & \multirow[t]{2}{*}{$\mathbf{P}$} & \multirow[t]{2}{*}{ Partial $\eta^{2}$} & \multirow[t]{2}{*}{$\mathbf{F}$} & \multirow{2}{*}{$\mathbf{P}$} & \multirow{2}{*}{ Partial $\eta^{2}$} \\
\hline & & & & & & & & & & \\
\hline \multirow{2}{*}{$\begin{array}{l}\text { Congru- } \\
\text { ent task }\end{array}$} & $\begin{array}{l}\text { Reaction } \\
\text { time }\end{array}$ & 66.72 & $\leq 0.001$ & 0.64 & 0.06 & 0.8 & 0.002 & 7.61 & 0.001 & 0.17 \\
\hline & Error & 57.99 & $\leq 0.001$ & 0.60 & 7.84 & 0.008 & 0.17 & 6 & 0.01 & 0.14 \\
\hline \multirow{2}{*}{$\begin{array}{l}\text { Incongru- } \\
\text { ent task }\end{array}$} & $\begin{array}{l}\text { Reaction } \\
\text { time }\end{array}$ & 43.08 & $\leq 0.001$ & 0.53 & 6.86 & 0.01 & 0.15 & 1.12 & 0.05 & 0.03 \\
\hline & Error & 2.05 & 0.16 & 0.05 & 8.46 & 0.006 & 0.18 & 2.94 & 0.08 & 0.07 \\
\hline \multicolumn{2}{|c|}{ Barthel index } & 0.09 & 0.8 & 0.002 & 0.03 & 0.9 & 0.001 & 8.7 & 0.06 & 0.19 \\
\hline
\end{tabular}


showed that resistance training focused on the upper limbs may enhance cognitive function in the elderly but we found no significant effect on daily living activities.

Evidence suggests that cognitive impairment may negatively affect the upper limb motor coordination [23] and dexterity $[9,24]$ in the elderly. This may be due to the disruption of the frontoparietal and frontostriatal networks, which in addition to cognition are also involved in the upper limb movements, and may be impaired during normal aging and the early stages of Alzheimer's disease [12]. The impact of executive functions on the daily activities of life, in which the upper limbs are involved has been suggested; however, the exact role of cognition and the quality of this relationship is not fully understood [24]. There is also growing evidence that physical activity can reduce the risk of Alzheimer's disease and dementia by almost $30 \%$ [25]. The exact underlying mechanism has not yet been determined. Some believe that exercise prevents and attenuates the risk factor of cardiovascular disorders, such as obesity and hypertension, which are also risk factors for dementia [25]. Others believe that physical exercise may improve cognitive function or prevent age-related cognitive decline directly through biological mechanisms, such as improving angiogenesis and neurogenesis, increasing plasticity in structures, such as the hippocampus, decreasing inflammation, increasing growth factors, and enhancing cerebrovascular reserve $[25,26]$. Also, different mechanisms underlying the benefits of resistance training on cognitive function have been suggested, including improving the transportation of oxygen and nutrients to brain areas involved in cognition, increasing growth factors (e.g., insulin-like growth factor-1 and neurotrophic factor), improved neurogenesis, and decreasing homocysteine [22, 27].

Our findings suggested that resistance training focused on the upper limbs may improve cognitive performance in older people. Although we did not find a study on the effectiveness of upper limb resistance training on cognitive function in the elderly, our findings were consistent with the results of most previous studies, which found positive effects of resistance training on cognitive function in the elderly [14, 22, 28-30]. But Ansai et al. [31] and Kimura et al. [32] did not find a significant difference in the cognitive function of the elderly who did resistance training compared to the control group. These discrepancies may be related to the low adherence of the participants to the training sessions in the first study and the high level of fitness in participants of the second study. In other words, the high level of fitness in the participants of the aforementioned study compared to the standard population caused a ceiling effect in the study sample, and if people with less fitness level were enrolled in the study, the effect of exercise on cognitive function could be more accurately determined.

In the present study, no significant difference was found between the two groups in daily living activities. This finding may be due to the better responsiveness of the Barthel index to show changes in longitudinal follow-up studies [33] but the duration of the intervention in this study was relatively short. On the other hand, the Barthel index is sensitive to changes in people with severe disabilities [34]. Therefore, to investigate the effects of upper limb resistance training on daily living activities, more clinical trials with more responsive and sensitive [35] outcome measures (e.g., functional independence Measure) are wanted.

Although as mentioned earlier, this is one of the first studies to evaluate the effectiveness of upper limb resistance training on cognitive function in the elderly, it has some limitations. First, the effects of resistance training on brain alterations were not investigated in this study. Also, only one cognitive outcome measure was used to evaluate the cognitive function and it is suggested that future studies examine the effectiveness of these exercises on diverse cognitive functions (e.g., attention and memory). In the present study, the effect of upper extremity resistance training in the elderly with normal cognitive function or with minimal cognitive deficits was investigated. It is suggested that in future studies, the effect of these exercises on the cognitive function of the elderly with cognitive deficits should be studied.

\section{Conclusion}

It seems that upper limbs resistance training may be efficacious in improving cognitive function in the elderly, and including these exercises in daily routines in addition to the physical and psychological effects also may have cognitive benefits.

\section{Ethical Considerations}

\section{Compliance with ethical guidelines}

All procedures were approved by the Ethics Committee of Shahid Beheshti University of Medical Sciences (IR.SBMU.RETECH.REC.1399.371) and all of the participants signed an informed consent form before their participation in the study. 


\section{Funding}

This research was funded and supported by school of Rehabilitation, Shahid Beheshti University of Medical Sciences.

\section{Authors' contributions}

Conceptualization: Navid Mirzakhani and Ali Asghar Jamebozorgi; Methodology: Navid Mirzakhani, Ali Asghar Jamebozorgi, Seyed Mehdi Tabatabaee, and Habib Esmaeilian; Investigation: Habib Esmaeilian; Data curation: Seyed Mehdi Tabatabaee; Writing - original draft: Mahnaz Hejazi-Shirmard and Navid Mirzakhani; Writing - review, and editing: Mahnaz Hejazi-Shirmard; Supervision: Navid Mirzakhani.

\section{Conflict of interest}

The authors declared no conflict of interests.

\section{Acknowledgments}

The authors would like to thank the Nursing Home staff and all participants in this study.

\section{References}

[1] Li Z, Peng X, Xiang W, Han J, Li K. The effect of resistance training on cognitive function in the older adults: A systematic review of randomized clinical trials. Aging Clinical and Experimental Research. 2018; 30(11):1259-73. [DOI:10.1007/ s40520-018-0998-6] [PMID]

[2] Bonder B, Dal Bello-Haas V. Functional performance in older adults. Philadelphia: FA Davis; 2017. https://www. google.com/books/edition/Functional_Performance_in_ $=0$

[3] Chang YK, Pan CY, Chen FT, Tsai CL, Huang CC. Effect of resistance-exercise training on cognitive function in healthy older adults: A review. Journal of Aging and Physical Activity. 2012; 20(4):497-517. [DOI:10.1123/japa.20.4.497] [PMID]

[4] Zaninotto P, Batty GD, Allerhand M, Deary IJ. Cognitive function trajectories and their determinants in older people: 8 years of follow-up in the English Longitudinal Study of Ageing. Journal of Epidemiology and Community Health. 2018; 72(8):685-94. [DOI:10.1136/jech-2017-210116] [PMID] [PMCID]

[5] Northey JM, Cherbuin N, Pumpa KL, Smee DJ, Rattray B. Exercise interventions for cognitive function in adults older than 50: A systematic review with meta-analysis. British Journal of Sports Medicine. 2018; 52(3):154-60. [DOI:10.1136/bjsports-2016-096587] [PMID]
[6] Karssemeijer EGA, Aaronson JA, Bossers WJ, Smits T, Olde Rikkert MGM, Kessels RPC. Positive effects of combined cognitive and physical exercise training on cognitive function in older adults with mild cognitive impairment or dementia: A meta-analysis. Ageing Research Reviews. 2017; 40:75-83. [DOI:10.1016/j.arr.2017.09.003] [PMID]

[7] Falck RS, Davis JC, Best JR, Crockett RA, Liu-Ambrose T. Impact of exercise training on physical and cognitive function among older adults: A systematic review and meta-analysis. Neurobiology of Aging. 2019; 79:119-30. [DOI:10.1016/j.neurobiolaging.2019.03.007] [PMID]

[8] Landrigan JF, Bell T, Crowe M, Clay OJ, Mirman D. Lifting cognition: A meta-analysis of effects of resistance exercise on cognition. Psychological Research. 2020; 84(5):1167-83. [DOI:10.1007/s00426-019-01145-x] [PMID]

[9] Bramell-Risberg E, Jarnlo GB, Elmståhl S. Slowing of alternating forearm movements is associated with cognitive impairment in community-dwelling older people. Dementia and Geriatric Cognitive Disorders. 2010; 29(5):457-66. [DOI:10.1159/000305093] [PMID]

[10] Hesseberg K, Tangen GG, Pripp AH, Bergland A. Associations between cognition and hand function in older people diagnosed with mild cognitive impairment or dementia Dementia and Geriatric Cognitive Disorders Extra. 2020; 10(3):195-204. [DOI:10.1159/000510382] [PMID] [PMCID]

[11] Kluger A, Gianutsos JG, Golomb J, Ferris SH, George AE, Franssen E, et al. Patterns of motor impairment in normal aging, mild cognitive decline, and early Alzheimer's disease. The Journals of Gerontology. Series B, Psychological Sciences and Social Sciences. 1997; 52B(1):P28-39. [DOI:10.1093/ geronb/52B.1.P28] [PMID]

[12] Scherder E, Dekker W, Eggermont L. Higher-level hand motor function in aging and (preclinical) dementia: Its relationship with (instrumental) activities of daily life-a mini-review. Gerontology. 2008; 54(6):333-41. [DOI:10.1159/000168203] [PMID]

[13] Seyedian M, Falah M, Nourouzian M, Nejat S, Delavar A, Ghasemzadeh H. [Validity of the Farsi version of mini-mental state examination (Persian)]. Journal of Medical Council of Iran. 2008; 25(4):408-14. http:/ /jmciri.ir/article-1-1170-fa.htm

[14] Cassilhas RC, Viana VA, Grassmann V, Santos RT, Santos RF, Tufik $S$, et al. The impact of resistance exercise on the cognitive function of the elderly. Medicine and Science in Sports and Exercise. 2007; 39(8):1401-7. [DOI:10.1249/ mss.0b013e318060111f] [PMID]

[15] Scarpina F, Tagini S. The stroop color and word test Frontiers in Psychology. 2017; 8:557. [DOI:10.3389/fpsyg.2017.00557] [PMID] [PMCID]

[16] Stroop JR. Studies of interference in serial verbal reactions. Journal of Experimental Psychology. 1935; 18(6):643-62 [DOI:10.1037/h0054651]

[17] Assef EC, Capovilla AG, Capovilla FC. Computerized stroop test to assess selective attention in children with attention deficit hyperactivity disorder. The Spanish Journal of Psychology. 2007; 10(1):33-40 [DOI:10.1017/S1138741600006296] [PMID]

[18] Zarghi A, Zali A, Tehranidost M, Ashrafi F, Zarindast M, Moazzezi M, et al. Assessment of selective attention with CSCWT (Computerized Stroop Color-Word Test) among children and adults. US-China Education Review. 2012; 1:121-7. https://eric.ed.gov/?id=ED530676 
[19] Wade D, Collin C. The Barthel ADL Index: A standard measure of physical disability? International Disability Studies. 1988; 10(2):64-7. [PMID]

[20] Oveisgharan S, Shirani S, Ghorbani A, Soltanzade A, Baghaei A, Hosseini S, et al. Barthel index in a Middle-East country: Translation, validity and reliability. Cerebrovascular Diseases. 2006; 22(5-6):350-4. [DOI:10.1159/000094850] [PMID]

[21] Hormozi S, Alizadeh-Khoei M, Sharifi F, Taati F, Aminalroaya $R$, Fadaee $S$, et al. Iranian version of barthel index: Validity and reliability in outpatients' elderly. International Journal of Preventive Medicine. 2019; 10:130. [DOI:10.4103/ ijpvm.IJPVM_579_18] [PMID] [PMCID]

[22] Anderson-Hanley C, Nimon JP, Westen SC. Cognitive health benefits of strengthening exercise for communitydwelling older adults. Journal of Clinical and Experimental Neuropsychology. 2010; 32(9):996-1001. [PMID]

[23] Schröter A, Mergl R, Bürger K, Hampel H, Möller HJ, Hegerl U. Kinematic analysis of handwriting movements in patients with Alzheimer's disease, mild cognitive impairment, depression and healthy subjects. Dementia and Geriatric Cognitive Disorders. 2003; 15(3):132-42. [DOI:10.1159/000068484] [PMID]

[24] Rodríguez-Aranda C, Mittner M, Vasylenko O. Association between executive functions, working memory, and manual dexterity in young and healthy older adults: An exploratory study. Perceptual and Motor Skills. 2016; 122(1):165-92. [DOI:10.1177/0031512516628370] [PMID]

[25] Tari AR, Norevik CS, Scrimgeour NR, Kobro-Flatmoen A, Storm-Mathisen J, Bergersen LH, et al. Are the neuroprotective effects of exercise training systemically mediated? Progress in Cardiovascular Diseases. 2019; 62(2):94-101. [DOI:10.1016/j.pcad.2019.02.003] [PMID]

[26] Quigley A, MacKay-Lyons M, Eskes G. Effects of exercise on cognitive performance in older adults: A narrative review of the evidence, possible biological mechanisms, and recommendations for exercise prescription. Journal of Aging Research. 2020; 2020:1407896. [DOI:10.1155/2020/1407896] [PMID] [PMCID]

[27] Tsai CL, Wang CH, Pan CY, Chen FC. The effects of longterm resistance exercise on the relationship between neurocognitive performance and GH, IGF-1, and homocysteine levels in the elderly. Frontiers in Behavioral Neuroscience. 2015; 9:23. [DOI:10.3389/fnbeh.2015.00023]

[28] Best JR, Chiu BK, Liang Hsu C, Nagamatsu LS, Liu-Ambrose $\mathrm{T}$. Long-term effects of resistance exercise training on cognition and brain volume in older women: Results from a randomized controlled trial. Journal of the International Neuropsychological Society. 2015; 21(10):745-56. [DOI:10.1017/ S1355617715000673] [PMID]

[29] Chupel MU, Direito F, Furtado GE, Minuzzi LG, Pedrosa FM, Colado JC, et al. Strength training decreases inflammation and increases cognition and physical fitness in older women with cognitive impairment. Frontiers in Physiology. 2017; 8:377. [DOI:10.3389/fphys.2017.00377] [PMID] [PMCID]

[30] Fragala MS, Beyer KS, Jajtner AR, Townsend JR, Pruna GJ, Boone $\mathrm{CH}$, et al. Resistance exercise may improve spatial awareness and visual reaction in older adults. Journal of Strength and Conditioning Research. 2014; 28(8):2079-87. [DOI:10.1519/JSC.0000000000000520] [PMID]
[31] Ansai JH, Rebelatto JR. Effect of two physical exercise protocols on cognition and depressive symptoms in oldest-old people: A randomized controlled trial. Geriatrics \& Gerontology International. 2015; 15(9):1127-34. [DOI:10.1111/ ggi.12411] [PMID]

[32] Kimura K, Obuchi S, Arai T, Nagasawa H, Shiba Y, Watanabe $S$, et al. The influence of short-term strength training on health-related quality of life and executive cognitive function. Journal of Physiological Anthropology. 2010; 29(3):95-101. [DOI:10.2114/jpa2.29.95] [PMID]

[33] Kwon S, Hartzema AG, Duncan PW, Min-Lai S. Disability measures in stroke: Relationship among the Barthel Index, the Functional Independence Measure, and the Modified Rankin Scale. Stroke. 2004; 35(4):918-23. [PMID]

[34] Lee SY, Kim DY, Sohn MK, Lee J, Lee SG, Shin YI, et al. Determining the cut-off score for the Modified Barthel Index and the Modified Rankin Scale for assessment of functional independence and residual disability after stroke. PLoS One 2020; 15(1):e0226324. [DOI:10.1371/journal.pone.0226324] [PMID] [PMCID]

[35] Dromerick AW, Edwards DF, Diringer MN. Sensitivity to changes in disability after stroke: A comparison of four scales useful in clinical trials. Journal of Rehabilitation Research and Development. 2003; 40(1):1-8. [PMID] 
This Page Intentionally Left Blank 